\title{
La concepción posthegeliana del reconocimiento en la filosofía de Judith Butler
}

\section{The post-Hegelian conception of recognition in Judith Butler'philosophy}

\author{
MILTON ABELLÓN \\ Universidad de Buenos Aires / CONICET
}

Recibido: 22/12/2019 Aceptado:03/08/2020

\section{RESUMEN}

Este trabajo muestra que, tras la publicación de Sujetos del deseo, Judith Butler elabora una concepción posthegeliana del reconocimiento que consta de una mediación social, basada en la noción hegeliana de «Eticidad», y una mediación normativa, apoyada en la idea foucaultiana del poder. Sostengo que dicha concepción implica dos sentidos de «sujeto» (i. figura históriconormativa y ii. resultado de un proceso de formación) que conforman el carácter sustituible y precario de la subjetividad. Finalmente, tras algunos señalamientos críticos sobre la interpretación butleriana de Hegel, indico la relevancia del tema estudiado el marco más general de su filosofía.

PALABRAS CLAVE

JUDITH BUTLER; RECONOCIMIENTO; MEDIACIÓN SOCIAL Y NORMATIVA; SUJETO; SUSTITUIBILIDAD Y PRECARIEDAD

\section{ABSTRACT:}

This work proves that, after Subjects of Desire, Judith Butler elaborates a post-Hegelian conception of recognition that consists of a social mediation, based on the Hegelian idea of «System of customs», and a normative mediation, based on the Foucaultian notion of power. I argue that this conception implies two senses of «subject» (i. A historical-normative figure and ii. The result of a process of becoming) that constitutes the substitutable and precarious 
nature of subjectivity. Finally, after some critical points about Hegel's Butlerian interpretation, I indicate the relevance of the subject studied in the more general framework of her philosophy. KEYWORDS

JUDITH BUTLER; RECOGNITION; SOCIAL AND NORMATIVE MEDIATION; SUBJECT; SUBSTITUTABILITY AND PRECARIOUSNESS

\section{INTRODUCCIÓN}

EL RECONOCIMIENTO ES UNA problemática que en las últimas décadas ha cobrado cada vez más protagonismo en los debates de la filosofía contemporánea. Fichte fue el primero en desarrollar la categoría de «reconocimiento» al otorgarle una especificidad propia en Fundamentos del derecho natural y en Ética. Sin embargo, la concepción sistemática de Hegel se estableció como el referente inmediato de las consideraciones contemporáneas.

Hegel aborda este tema en sus textos de juventud y madurez. Entre los primeros se destacan Sistema de la eticidad, Esbozos de Sistema de la época de Jena (1803-1804 / 1805-1806) y Fenomenología del espíritu (1807). Entre los segundos, Enciclopedia de las ciencias filosóficas y Principios de la filosofía del derecho. Si bien estas obras tienen sus propias peculiaridades y la Hegelforschung se debate sobre las rupturas y continuidades entre ellas, puede afirmarse que el reconocimiento es uno de los principios fundamentales de la filosofía práctica de Hegel, puesto que constituye un movimiento ineludible de la realización plena de la autoconciencia intersubjetiva (Düsing 1986, Siep 2006). Dicho movimiento incluye no solo diversas figuras de la conciencia, sino también distintos estadios de la historia cultural de la humanidad y múltiples estructuras de relaciones intersubjetivas e institucionales que culminan en los vínculos jurídicos y sociales del Estado constitucional moderno europeo y postrevolucionario (De la Maza 2009, Theunissen 1982).

El reconocimiento se establece como un problema fundamental de la filosofía contemporánea sobre todo a partir de 1930, gracias al lugar privilegiado que Kojève le otorga en sus famosos cursos sobre Hegel dictados en la École des Hautes Études. En 1990, se instala la cuestión de la «política del reconocimiento», que debe su nombre al texto homónimo de Charles Taylor ([1992] 1997), donde el autor considera al reconocimiento intersubjetivo como la condición de la realización de la identidad individual. Pero más allá del desarrollo de Taylor, lo más relevante para los debates contemporáneos es el nuevo espacio problemático que abre. A partir de esa fecha, el tema del reconocimiento no se limita ya a las clásicas relaciones interpersonales y sociales, sino que involucra otras cuestiones relativas a la interculturalidad 
y la multiculturalidad, a las políticas de la identidad y la diferencia, a la globalización, a la justicia social y distributiva, y a cuestiones ético-políticas de diversa índole. En el siglo XXI existe un campo de múltiples lineamientos teóricos y variados debates en torno al reconocimiento. Actualmente la clásica discusión sobre la formación de la identidad subjetiva y la justicia -en la que se destaca, entre otros, el desarrollo de Honneth- convive con las posiciones normativistas norteamericanas y con las discusiones bioéticas acerca de la autonomía personal. ${ }^{1}$

La apropiación que Judith Butler realiza de algunas ideas de Hegel para pensar el problema del reconocimiento se ubica dentro de la llamada «política del reconocimiento» y constituye gran parte de su corpus bibliográfico. Su recepción de Hegel, a diferencia de la tendencia analítica y la pragmática imperantes en Estados Unidos, se realiza por el movimiento fenomenológico que cobra notoriedad en dicho país a partir de 1980 (Rockmore 2005). A mediados de esta década, publica la versión revisada y ampliada de su tesis doctoral, Sujetos del deseo, en la que estudia las nociones de «deseo» (Begierde / desire) y «reconocimiento» en el Capítulo IV la Fenomenología y algunas de sus recepciones francesas. Esta obra es relevante porque establece los lineamientos generales de su interpretación de Hegel y sienta las bases de su posterior concepción posthegeliana del reconocimiento.

La apropiación butleriana de la noción hegeliana del reconocimiento ha recibido poca atención por parte de los estudios especializados. Hasta el momento contamos con escasos trabajos que consideran de manera específica el tema. Dentro de los estudios butlerianos $-s c$. aquellos especializados en el pensamiento de Butler-, se encuentran los textos de Lloyd (2007), Ong-VanCung (2010) y Stark (2014). Dentro de la Hegelforschung interesada en las recepciones contemporáneas, se destacan los trabajos de Assalone (2015b), Bouton (2009) y De la Maza (2010). Todas estas investigaciones enfatizan el aspecto normativo de la concepción butleriana del reconocimiento, revelando un punto clave de la cuestión que ocupa al presente trabajo. Sin embargo, dichas investigaciones cuentan con dos insuficiencias importantes. La primera es que realizan un abordaje del corpus butleriano muy limitado. Ninguna considera la totalidad de las obras en las que Butler examina el reconocimiento. Esto tiene como consecuencia que no contemos aún con un texto sistemático de referencia sobre el tema. Además, conlleva la desatención de ciertos movimientos conceptuales que la filósofa realiza a lo largo de sus obras. La segunda insuficiencia consiste en que no determinan con claridad qué aspectos de la noción hegeliana del reconocimiento retoma Butler. En

$1 C f$. Assalone (2015a), De la Maza (2010), Siep (2004, 2010, 2014). 
este sentido, a la limitación del corpus considerado se suma cierta falta de especificidad conceptual.

En lo que sigue, desarrollo de manera sistemática, y desde mi propia hermenéutica del pensamiento de Butler, su concepción posthegeliana del reconocimiento. ${ }^{2}$ Sostengo que ésta consta de dos dimensiones íntimamente relacionadas: la mediación social del reconocimiento, basada en su apropiación de la noción hegeliana de Eticidad (Sittlichkeit), y la mediación normativa, desarrollada a partir de su apropiación de la concepción del poder de Foucault. ${ }^{3}$ Para ello, en primer lugar, repongo brevemente las ideas principales de la hermenéutica del Capítulo IV de Fenomenología (Parte introductoria y Sección A) que Butler desarrolla en Sujetos del deseo. ${ }^{4}$ Realizo esto por dos razones: (a) porque sus claves interpretativas son retomadas, aunque desde otra perspectiva, en su posterior reconsideración del reconocimiento, y (b) porque la conclusión a la que allí llega constituye el punto de partida de su concepción posthegeliana del reconocimiento. En segundo lugar, expongo las mediaciones antedichas y pongo de relieve las dos nociones de «sujeto» que les son concomitantes. En tercer lugar, me ocupo del carácter sustituible y precario de la subjetividad implicado por las mediaciones. Finalmente, en cuarto lugar, realizo algunos señalamientos críticos sobre la interpretación butleriana de Hegel e indico la relevancia que tiene el tema estudiado en el marco más general de su filosofía.

\section{BREVIARIO DE LA LECTURA BUTLERIANA DEL CAPÍTULO IV DE LA FENOMENOLOGÍA}

El problema del Capítulo IV de la Fenomenología, que expone la figura de la Autoconciencia, es la realización de la verdadera unidad de la autoconciencia consigo misma. Para alcanzar tal unidad, la autoconciencia, que en principio persiste como conciencia, debe superar la contraposición con su objeto. Pero dado que ella es, de primeras, una figura autónoma para sí, busca también -en el curso de su movimiento- afirmar su autonomía respecto de su objeto a través de diversas referencias negativas hacia él.

2 Mi objetivo no es establecer los vínculos entre la posición de Butler y otras apropiaciones contemporáneas de Hegel. Ello requiere otro tipo de trabajo y excede los límites del presente texto. Parte de tal objetivo fue considerado por Assalone (2015b), Bouton (2009) y De la Maza (2010). El propósito es problematizar y profundizar el estudio del posicionamiento de Butler, intentando saldar las deudas e insuficiencias de la literatura especializada, de manera tal de poder contribuir al corpus bibliográfico con un texto que pueda funcionar de referencia sobre el tema.

3 Butler no usa «mediaciones», término que retomo de Assalone (2015b) y con el que me refiero a las determinaciones esenciales del sujeto implicadas en su proceso de formación a través del reconocimiento.

4 Esta cuestión fue desarrollada in extenso en Abellón (2020a). 
La primera de tales referencias se expone en la Parte introductoria del capítulo. Aquí, el objeto de la autoconciencia es la Vida en tanto que «algo vivo» (Lebendiges) - y la referencia negativa es la apetencia (Begierde) (Hegel 2010, 104). ${ }^{5}$ Esta última es una referencia negativa e inmediata, y consiste en el acto de aniquilar y consumir las cosas vivas independientes. Pero este movimiento, por el que la autoconciencia busca probar su autonomía respecto de su objeto, fracasa porque la apetencia precisa de la existencia del objeto a aniquilar, engendrándolo de nuevo y persistiendo ella misma como tal.

El tema del reconocimiento se introduce tras el fracaso de la apetencia, específicamente en el marco de la duplicación de la autoconciencia. El reconocimiento recíproco es el momento en que queda acabado el concepto de la autoconciencia. Se trata de la unidad espiritual de la duplicación, unidad en la cual ambas autoconciencias alcanzan la verdad de su autonomía en el seno de la mediación intersubjetiva que se corresponde con la estructura del Espíritu: «yo que es nosotros, y nosotros que es yo» (ibid., 108; el subrayado es del original).

Pero el movimiento del reconocer, según se desarrolla en la Sección A del capítulo, consta de dos momentos que fracasan en el logro de la autonomía y la unidad mencionadas. El primero es la lucha a vida o muerte, que revela que ambas autoconciencias deben vivir para poder ser reconocidas. El segundo es la dominación y la servidumbre, por el que sólo se alcanza un reconocimiento desigual y unilateral, al tiempo que la libertad del siervo se revela finalmente como obstinación.

La lectura que Butler expone en Sujetos del deseo toma dos claves interpretativas que son retomadas en Mecanismos psíquicos (1997) y Sois mon corps (2010), textos que continúan el desarrollo de la hermenéutica iniciada en la primera obra.

La primera clave interpretativa es el deseo (desire), apetencia espiritualizada que, tras el fracaso de la Begierde y en el contexto de la duplicación, se reconfigura como «deseo de reconocimiento», deseo propiamente humano. ${ }^{6}$ La satisfacción de este último no radica ya en la mera aniquilación del objeto, sino en el logro del hacer duplicado por el que cada autoconciencia encontraría el reflejo de sí en la otra.

5 Las referencias de la Fenomenología se realizan según la numeración canónica de la traducción castellana de la edición bilingüe de Gómez Ramos (Abada, 2010).

6 «Begierde» recibió dos traducciones en las ediciones castellanas de la Fenomenología. Roces tradujo el término por «apetencia» (2007, p. 108) y Gómez Ramos por «deseo» (2010, 247). En la edición inglesa de Miller, que usa Butler, también aparece traducido por «desire» (1977, § 167). Cabe señalar que «deseo de reconocimiento» no es un término de Hegel sino una expresión acuñada por Kojève en sus cursos sobre la Fenomenología ([1947] 2006, p. 13). 
La segunda clave hermenéutica es el cuerpo (body - corps).$^{7}$ En Sois mon corps, Butler sostiene que la noción hegeliana de «figura» (Gestalt) puede entenderse en términos corporales o, más precisamente, que el cuerpo es uno de los modelos fundamentales para pensar la noción de figura en el Capítulo IV. Su tesis específica es que las autoconciencias, figuras autónomas (selbständige Gestalten) del medio vital universal, son formas (formes) o cuerpos vivos (corps vivant). ${ }^{8}$ En este sentido, la objetualidad de la vida se reconfigura en términos corporales en tanto el cuerpo representa la objetividad vital de la autoconciencia.

Según Butler, el cuerpo es la forma que, por un lado, constituye la determinidad y autonomía de cada una de las autoconciencias en tanto que figuras autónomas y subsistencias para sí. Por otro lado, es también aquello que revela su carácter extático, su «ser fuera de sí». Al estar en relación de enfrentamiento mutuo, cada autoconciencia experimenta que su esencia no es una propiedad exclusiva, pues la encuentra también fuera de sí, en la otra autoconciencia en tanto que yo corporal independiente. A esto se refiere Butler al hablar de la sustituibilidad (substitutability /substituabilité) de la autoconciencia: la aparición en escena de otro individuo amenaza la autonomía de cada autoconciencia al hacer manifiesto que el yo está objetivado en otro cuerpo vivo. Esto trae aparejado una experiencia mutua de extrañamiento que el reconocimiento recíproco, como unidad de la duplicación, vendría a superar.

Desde este posicionamiento general y a partir de estas claves interpretativas, Butler caracteriza la lucha a vida o muerte como un «erotismo anti-coporal», por el que el deseo de alcanzar la autonomía busca satisfacerse mediante la aniquilación del cuerpo ajeno ([1987] 2012, p. 95). Ante el fracaso de este momento y la necesidad de que ambas conciencias se mantengan con vida, la dominación constituye la espiritualización del intento de matar: el señor niega la libertad y la autonomía del siervo convirtiéndolo en un mero instrumento. Este movimiento de dominación se realiza mediante un imperativo de sustitución corporal que reza así: «sé tú mi cuerpo para mí» ([1997] 2015, p. 48). ${ }^{9}$ Se trataría de una orden que el señor dirige implícitamente al siervo y que lo obliga a ser el cuerpo que él ( $s c$. el señor) rechaza para sí mismo, puesto que lo mantiene dependiente del elemento vital de la existencia, dependencia que

7 La corporalidad es una clave hermenéutica externa al Capítulo IV, puesto aquí no aparecen ni «Körper» ni «Leib», ni sus cognados.

8 En la traducción del Capítulo IV de la Fenomenología que aparece al final de Sois mon corps (2010, pp. 107-147), «Gestalt» se traduce por «figure». En la versión inglesa que se publica en Companion to Hegel (2011, pp. 611-640), se traduce por «shape», al igual que en la traducción de Miller. Para este punto de la interpretación de Butler véase Sabot (2011).

9 El imperativo se formula también en Sujetos del deseo ([1987] 2012, p. 96) y en Sois mon corps $(2010$, p. 83). 
contrasta con la búsqueda afirmativa de su autonomía en tanto que conciencia pura. A través de tal imperativo, el siervo queda reducido a un mero cuerpo, un instrumento sin vida que, por medio del servicio y del trabajo, media al señor consigo mismo y con las cosas naturales.

El desarrollo paradójico de esta dialéctica muestra la inversión de la dominación en servidumbre y revela la libertad del siervo como obstinación. Pero lo que interesa aquí no es la exposición de estas inversiones, sino la conclusión de Butler. Al final del Capítulo I de Sujetos del deseo, se afirma que lo que muestra la dialéctica de la dominación y la servidumbre a propósito del reconocimiento recíproco es la insuficiencia de la escena diádica. La idea de Butler es que el reconocimiento recíproco sólo puede realizarse en el seno de su compleja dimensión social e histórica. Esto último constituye el punto de partida de su concepción posthegeliana del reconocimiento, que será desarrollada a continuación.

\section{LA CONCEPCIÓN POSTHEGELIANA DEL RECONOCIMIENTO}

En Dar cuenta de sí(2005), Butler se propone realizar una «caracterización posthegeliana del reconocimiento que procura establecer la base social del mismo» ([2005] 2009, p. 34). Entiendo que este propósito no se reduce a esta obra. En textos anteriores y posteriores, Butler trabaja el problema del reconocimiento desde una perspectiva contemporánea, desarrollando una concepción posthegeliana con rasgos neohegelianos. Ya en Mecanismos psíquicos se rastrean claras elaboraciones en esta dirección. En lo que atañe a los escritos del nuevo milenio, se destacan Deshacer el género (2004), Vida precaria (2004), Marcos de guerra (2009) y Desposesión (2013). Por otro lado, hay un conjunto de textos en los que se realizan menciones importantes sobre el reconocimiento, aunque este no sea el tema central que abordan. En este segundo grupo de obras se encuentran Contingencia, hegemonía y universalidad (2000), El grito de Antígona (2000) y Cuerpos aliados (2015).

La idea de cuño hegeliano que a la que Butler adhiere desde Sujetos del deseo y que constituye la base de su concepción posthegeliana del reconocimiento es que este último es la condición esencial de la formación y realización del sujeto. Ahora bien, dada la insuficiencia de la escena diádica, es preciso considerar otros aspectos del reconocimiento, que atañen a la constitución cultural de la subjetividad. En este sentido, según mi interpretación, la concepción butleriana del reconocimiento consta de dos dimensiones: (i) la mediación social, basada en su apropiación de la idea de Eticidad de Hegel, y (ii) la mediación normativa, basada en su apropiación de la concepción del poder de Foucault. ${ }^{10}$ En lo que sigue, desarrollo ambas

10 La distinción entre estas mediaciones no debe considerarse en sentido fuerte. Desde una 
mediaciones a partir del tratamiento conjunto de las obras de Butler, aunque sin perder las especificidades que ellas presentan.

\section{III.1. LA MEDIACIÓN SOCIAL DEL RECONOCIMIENTO}

La noción hegeliana de Eticidad (Sittlichkeit) ofrece, según Butler, «una versión social de las normas en virtud de las cuales el reconocimiento recíproco podría sostenerse de manera más estable de lo que supondrían la lucha a vida o muerte o el sistema de servidumbre» ([2005] 2009, p. 46). ${ }^{11}$ Aunque Hegel aborda la Eticidad en varios lugares de su corpus, Butler se detiene principalmente en algunos aspectos de la exposición del Capítulo $\mathrm{V}$ de la Fenomenología, específicamente de «La realización efectiva de la autoconciencia racional por medio de sí misma». En esta sección, que conforma el segundo momento de la Razón, se abre el «reino de la eticidad» por medio del concepto de la autoconciencia reconocida, teniendo lugar la realización inmediata del Espíritu, es decir, la realización inmediata y efectiva de la autoconciencia intersubjetiva en el sistema de costumbres de un pueblo. Al respecto, Hegel sostiene:

Pues ésta [sc. la Eticidad] no es [...] otra cosa que la absoluta unidad espiritual de la esencia de los individuos en el seno de la realidad efectiva autónoma de estos; una autoconciencia universal [...] Esta unidad [...], esta substancia universal habla su lengua universal (allgemeine Sprache) en las costumbres y las leyes (Gesetzen) del pueblo de este individuo; pero esta esencia [...] no es otra cosa que la expresión de la individualidad singular misma, aparentemente contrapuesta a esta substancia; las leyes enuncian lo que cada individuo singular es y hace; el individuo no sólo las reconoce como su cosidad objetual universal, sino también, en la misma medida, se reconoce en ésta, o bien, se reconoce como singularizado en su propia individualidad y en cada uno de sus conciudadanos [...] Por tanto, en el espíritu universal, cada uno [...] está tan cierto de los otros como de sí

perspectiva contemporánea, Butler encuentra ya en la idea hegeliana de Eticidad ciertos aspectos normativos. Sin embargo, al referirse a ella busca iluminar el carácter social e intersubjetivo del reconocimiento. Por el contrario, al incorporar el planteo de Foucault, la filósofa pretende enfatizar la normatividad social que produce al sujeto a través del reconocimiento. En última instancia, se trata de dos aspectos de una misma cuestión, puesto que -para Butler- lo social es necesariamente normativo. El tratamiento que Butler realiza de Hegel y Foucault en este contexto es netamente apropiativo; esto quiere decir que se sirve de algunas de sus ideas para pensar el problema del reconocimiento desde su propia perspectiva.

11 De los textos especializados, solo el de Bouton menciona la influencia de la idea de Eticidad en la concepción butleriana del reconocimiento (2009, pp. 58-59, 66-67). Sin embargo, el autor entiende y expone tal influencia en términos estatales, mientras Butler aclara expresamente en Contingencia, hegemonía y universalidad que, para ella, lo esencial de tal noción radica sólo en su aspecto socio-cultural ([2000] 2011, p. 176). 
[...] Los veo a ellos como a mí, y a mí como a ellos ([1807] 2010, 194-195; el subrayado es del original).

Butler encuentra en esta caracterización de la Eticidad la versión hegeliana de la tesis contemporánea según la cual el reconocimiento recíproco se realiza dentro de un marco cultural -instituido por la reiteración de las prácticas consuetudinarias y hegemónicas de los individuos-12 que media los intercambios intersubjetivos. En Contingencia, hegemonía y universalidad, afirma que la «Eticidad» designa un «conjunto de normas, convenciones y valores compartidos que constituyen el horizonte cultural en el cual el sujeto emerge a la autoconciencia», es decir, «un mundo cultural» que «media la relación del sujeto consigo mismo» ([2000] 2011, p. 176; el subrayado es mío). Esto quiere decir que la mediación del sujeto consigo mismo se alcanza a través de la mediación social que establece la Eticidad, definida en Cuerpos aliados precisamente como la «forma mediadora» de la individualidad ([2015] 2017, p. 215). La mediación social, en efecto, implica una serie de referencias subjetivas más amplia y compleja que la que se establecía en la dominación y la servidumbre. Por un lado, cada individuo se refiere a otros individuos. Por otro lado, cada uno se refiere también a la «sustancia ética» o sistema de costumbres, es decir, a la realidad social compartida en y a través de la cual los individuos se reconocen recíprocamente. El reconocimiento así alcanzado realiza, entonces, la mediación intersubjetiva del sujeto en el marco de la autoconciencia universal o colectiva de un sistema cultural determinado. ${ }^{13}$

Ahora bien, desde la perspectiva contemporánea de Butler, la concepción hegeliana del reconocimiento es insuficiente. Su «crítica epistemológica» (Stark 2014, p. 93-94) es que tal concepción presupone la reciprocidad intersubjetiva; presuposición que, además de funcionar como garantía del reconocimiento, no da cuenta de las relaciones de poder, de la normatividad constitutiva de la reconocibilidad (recognizability). Para dar cuenta de ella, Butler recurre a la concepción foucaultiana del poder. Su propuesta es «conducir el argumento hegeliano en una dirección foucaultiana», para mostrar que las mismas normas del reconocimiento producen el sujeto reconocible ([2004] 2006, p. 55). Según mi interpretación, Butler reconsidera la idea hegeliana de Eticidad en términos

12 Esta es una idea que Butler sostiene expresamente en Contingencia, hegemonía y universalidad ([2000] 2011, p. 28) y en Dar cuenta de si ([2005] 2009, pp. 30, 39), donde puede encontrarse una caracterización del marco cultural mediador como una universalidad social no trascendental. Por esta razón, me diferencio de la lectura de Malabou (2011, p. 18), quien sostiene que dicha universalidad constituye la dimensión trascendental del yo.

13 En la misma línea y siguiendo explícitamente a Hegel, Butler define «lo ético» en Desposesión como la interdependencia entre los individuos y las instituciones ([2013] 2017, pp. 134-135). 
normativo-foucaultianos. Desde esta perspectiva, el sistema de costumbres o marco cultural que media el reconocimiento intersubjetivo está conformado por un conjunto de normas que determinan históricamente aquello que es y no es reconocible como sujeto. ${ }^{14}$ En este sentido, afirma que el planteo normativo de Foucault es el «complemento (supplement) a Hegel» ([2005] 2009, p. 47), cuestión que será desarrollada en lo que sigue.

\section{2. LA MEDIACIÓN NORMATIVA DEL RECONOCIMIENTO}

La normatividad del reconocimiento se explica por dos cuestiones íntimamente relacionadas. La primera cuestión, presentada ya al final del apartado anterior, consiste en el carácter normativo del marco social que media el reconocimiento al determinar los criterios de reconocibilidad subjetiva. En Marcos de guerra, caracteriza las matrices normativas como «marcos epistémicos» que generan ontologías históricas de lo humano, y elabora ciertas distinciones conceptuales que permiten diferenciar niveles epistemológicos del reconocimiento y de su normatividad ([2009] 2010, pp. 13, 18-21).

La primera distinción es entre el «reconocimiento»y la «reconocibilidad». El primero es el acto recíproco entre dos o más individuos por el que éstos se re-conocen qua sujetos. La segunda es la condición de posibilidad del primero y consiste en el conjunto de normas, convenciones y términos que moldean el reconocimiento, haciendo posible que un individuo se convierta en un sujeto reconocible. La segunda distinción conceptual diferencia la «reconocibilidad» recién definida de la «inteligibilidad». Esta última es el esquema histórico que establece el ámbito de lo conocible y condiciona y precede a la reconocibilidad en tanto que a priori histórico. Finalmente, la tercera diferencia se establece entre el reconocimiento y el acto de aprehender (apprehending). Este último consiste en «el marcar, registrar o reconocer sin pleno reconocimiento» (ibid., p. 18). Se trata de una forma débil del conocer asociada al sentir o al percibir, que está facilitada pero no limitada por los esquemas de inteligibilidad y reconocibilidad. Por medio de la aprehensión es posible percatarse de que una vida humana está excluida de los criterios de reconocibilidad subjetiva.

A partir de lo dicho, pueden diferenciarse tres niveles epistemológicos de la normatividad del marco social mediador. El primero es el carácter normativo de los esquemas de inteligibilidad y reconocibilidad. El segundo radica en la normatividad del reconocimiento, la cual halla su fundamento en

14 En el mismo sentido, Butler define el marco social mediador en Vida precaria como un «campo diferencial de poder» que produce «concepciones normativas de lo humano» al establecer históricamente los criterios de reconocibilidad subjetiva ([2004] 2009, pp. 17, 72). Para una consideración similar véase Butler ([2000] 2001, pp. 17, 29-32). 
el primer nivel. El tercero concierne al carácter normativo del campo de la ininteligibilidad subjetiva, normatividad condicionada pero no limitada por el primero de los niveles.

Estos niveles epistemológicos están esencialmente relacionados con la segunda cuestión que explica la normatividad del reconocimiento. Se trata de la tesis que afirma que la sujeción normativa es inherente a la subjetivación del individuo, idea que Butler explica en Mecanismos. Siguiendo las consideraciones de Foucault sobre los regímenes de poder ${ }^{15}$, Butler distingue tres sentidos de «sujeción» que se unifican en la tesis mencionada. El primero es el sentido activo y consiste en el acto de «sujetar a». El segundo es el sentido pasivo, según el cual el sujeto es un ser sujetado, «sujeto a». El tercero es la «subjetivación»: el proceso de «devenir sujeto» ([1997] 2015, pp. 12-13). ${ }^{16} \mathrm{La}$ sujeción como subjetivación significa que el individuo se hace sujeto, se forma como tal, en y mediante la sujeción al poder, $s c$. a los esquemas normativos de inteligibilidad y reconocibilidad subjetivas, en los términos de Marcos de guerra. Al respecto, agrega:

El «sujeto» es presentado a menudo como si fuese intercambiable con «la persona»o «el individuo». Sin embargo, [...] el sujeto, más que identificarse de manera estricta con el individuo, debe entenderse como una categoría lingüística, un comodín (placeholder), una estructura en formación. Los individuos llegan a ocupar el lugar del sujeto y adquieren inteligibilidad sólo en tanto que están, por así decir, previamente establecidos en el lenguaje [sc. en los esquemas normativos de inteligibilidad y de reconocibilidad] [...] Ningún individuo deviene sujeto sin antes padecer sujeción o experimentar «subjetivación» ([1997] 2015, pp. 21-22).

15 Las obras de Foucault que Butler considera son: Vigilar y castigar (1986), Historia de la sexualidad I-II $(2002,2003)$ y Power/Knowledge: Selected Interviews and Other Writings, 1972-77 (1980). También considera, aunque en menor medida, la concepción de Althusser de la producción del sujeto por medio de la interpelación, basándose en Ideología y aparatos ideológicos del Estado (2012). Para la lectura butleriana de Althusser véase Davis (2012).

16 Estos tres sentidos de «sujeción» son retomados casi literalmente en la introducción de Los sentidos del sujeto ([2015] 2016, pp. 15-18). Lo que Butler entiende por «poder» en Mecanismos son las normas sociales subjetivantes, aquellas que delimitan históricamente lo que el sujeto es, al establecer los esquemas de inteligibilidad y reconocibilidad subjetivas. En Mecanismos, Butler no había elaborado aún las distinciones epistemológicas de Marcos de guerra. Sin embargo, esto no trae aparejado un problema conceptual dado que aquellas no son más que una elaboración sofisticada de ideas y nociones anteriores. Por ejemplo, las ideas de «poder» y «normas de reconocimiento» de Mecanismos aparecen conceptualizadas en Marcos de guerra bajo los conceptos de «inteligibilidad»y «reconocibilidad». En este sentido, es posible establecer un vínculo conceptual directo entre ambas obras. 
A partir de los tres sentidos de «sujeción» y de la diferenciación entre «sujeto» e «individuo», se pueden distinguir, según mi interpretación, dos sentidos de «sujeto». Según un primer sentido, correspondiente a lo que en la cita se caracteriza como «estructura en formación», el Sujeto es la figura de lo humano que el marco socio-cultural establece históricamente a partir de sus esquemas de inteligibilidad y reconocibilidad subjetivas. Según un segundo sentido, «sujeto» es el resultado del proceso de subjetivación. Bajo esta acepción, son «sujetos» aquellos individuos que devinieron tales por medio de la sujeción normativa y el reconocimiento intersubjetivo por ella posibilitado. La sujeción normativa es la condición del reconocimiento intersubjetivo y éste es el que confiere acabadamente al individuo el estatus social de sujeto. Adquirir este estatus significa habitar el «ámbito de la socialidad vivible» (Butler ([1997] 2015, p. 32), es decir, gozar de «vida social» (ibid., p. 39) o «existencia social» (ibid., p. 40). Por esta razón, Butler entiende que el «deseo por la existencia social» (ibid., p. 32) es concomitante al «deseo por la norma» (ibid., p. 32). Por el contrario, los individuos no sujetados normativamente quedan excluidos de la categoría de sujeto y, por lo tanto, relegados al ámbito de la ininteligibilidad cultural y de la «muerte social» (Butler [2009] 2010, p. 69). ${ }^{17}$ Estos individuos son aquellos que por no haberse subordinado a los esquemas normativos de inteligibilidad y reconocibilidad, no son reconocibles como sujetos, aunque sí susceptibles de ser aprehendidos como seres abyectos.

\section{3. SUSTITUBILIDAD, PRECARIEDAD Y PRECARIDAD}

La concepción posthegeliana del reconocimiento reconfigura también la cuestión de la sustituibilidad subjetiva que estaba presente en la hermenéutica butleriana del Capítulo IV de la Fenomenología. Butler introduce esta problemática en Dar cuenta de sí a través de su crítica a la objeción que Cavarero (1997) formula al modelo hegeliano del reconocimiento. Según reconstruye Butler, para Cavarero el límite del reconocimiento recíproco radica en el carácter personal y único de los individuos, por el cual no pueden identificarse como un «nosotros», es decir, como una identidad intersubjetiva y colectiva. Para Butler, esta objeción es desacertada y el límite mencionado es imposible, dado el carácter esencialmente extático y sustituible del sujeto. En una clara aproximación foucaultiana de Hegel, se pregunta: «¿Acaso la teoría social derivada de Hegel [sc. la Eticidad], con su insistencia en la perspectiva impersonal de la norma, replica estableciendo mi sustituibilidad?» ([2005] 2009 , p. 51). Su respuesta afirmativa se articula en dos argumentos generales.

El primero de ellos consiste en el carácter impersonal de los esquemas de inteligibilidad y de reconocibilidad subjetivas. Las normas de reconoci-

17 El término «muerte social» aparece también en Butler ([2013] 2017, p. 35). 
bilidad, a las que los individuos deben subordinarse para poder ser reconocibles qua sujetos, son impersonales por el hecho de ser sociales: ellas superan la mera perspectiva personal de los individuos porque son la obra del hacer hegemónico y no el resultado de un mero hacer individual. Por esta razón, la sujeción normativa concomitante a la subjetivación implica el paso de la perspectiva personal del individuo a la dimensión impersonal de las normas (De la Maza 2010, pp. 82-83; Mills 2015, pp. 51-52). En este sentido, Butler sostiene que «en cierta medida tendré que llegar a ser sustituible para hacerme reconocible» ([2005] 2009, p. 56).

El segundo argumento concierne al éxtasis subjetivo que pone de manifiesto la corporalidad. Al igual que en su hermenéutica del Capítulo IV de la Fenomenología, Butler sostiene que el cuerpo es la forma que otorga determinidad a cada individuo, pero también es la forma que manifiesta el éxtasis del sujeto en tanto revela su ser fuera de sí, exposición que pone de relieve la sustituibilidad subjetiva. En el marco de su caracterización posthegeliana del reconocimiento, el éxtasis y la sustituibilidad subjetivas no se reducen ya a la escena diádica, sino que remiten más bien al carácter social de todo individuo en tanto que ser corporal; cuestión que en Marcos de guerra se conceptualiza en los términos de una «ontología del cuerpo» en tanto que «ontología social» ([2009] 2010, p. 15). La idea central de Butler es que el éxtasis que caracteriza al individuo en tanto que este-singularcorporal es también un hecho universal, una condición compartida por todos los individuos que «reinstala el "nosotros"» y que «establece una estructura de sustituibilidad en el corazón de la singularidad» ([2005] 2009, p. 53). A propósito de esto, agrega:

Por mucho que cada uno de nosotros ambicione el reconocimiento y lo requiera, no por ello somos lo mismo que el otro [...] La singularidad del otro queda expuesta ante mí, pero la mía también se expone ante él. Esto no significa que seamos lo mismo; sólo quiere decir que estamos unidos a otro por lo que nos diferencia, a saber: nuestra singularidad. Con mucha frecuencia, el concepto de singularidad queda atado al romanticismo existencial y a una pretensión de autenticidad, pero entiendo que, justamente por carecer de contenido (without content), mi singularidad tiene algunas propiedades en común con la tuya y, por tanto, es en alguna medida un término sustituible [...] Hegel analiza el «esto» en la Fenomenología, donde puntualiza que nunca especifica sin generalizar y que el término, en su sustituibilidad misma, socava la especificidad que procura señalar: "Cuando digo: "una sola cosa", en realidad, digo lo que es desde un punto de vista completamente universal, puesto todo es una sola cosa; y, del mismo modo, "esta cosa" es todo lo que ustedes quieran. Si la describimos con mayor exactitud como "este pedazo de papel", entonces, cada uno de todos los 
pedazos de papel son "este pedazo de papel", y no he hecho sino enunciar el universal en todo momento» (Ibid., pp. 52-53).

La sustituibilidad, entonces, no se restringe a la esencia interna del individuo, sino que involucra también el aspecto universal de la singularidad, aquello que en Contingencia, hegemonía y universalidad aparece conceptualizado como sensibilidad y que involucra el sentir, el percibir y toda peculiaridad de la naturaleza. ${ }^{18}$

Esta concepción de la sustituibilidad subjetiva constituye lo que Butler denomina «precariedad», concepto que se corresponde con un primer sentido de «desposesión» y con la idea de «vulnerabilidad primaria». ${ }^{19}$ La precariedad es una condición existencial de la vida humana que consiste en el carácter originariamente relacional de los individuos, y en la dependencia social y normativa que les es esencial por estar implicada necesariamente en el proceso de subjetivación y desubjetivación. Se trata de la violencia primaria que padecen todos los individuos en tanto su formación subjetiva está sujeta a una necesaria sumisión normativa y social. Según Butler, «la precariedad subraya nuestra radical sustituibilidad» porque el cuerpo, al manifestar el éxtasis relacional de la vida intersubjetiva, constituye el indicador por excelencia de la nuestra vulnerabilidad primaria ([2009] 2010, p. 31).

Ahora bien, la desposesión, además de su dimensión existencial, tiene una dimensión política, que se corresponde con la noción de «precaridad», la cual tiene a la precariedad como su condición de posibilidad. La precaridad es la distribución efectiva de la precariedad: el repudio y el rechazo efectivos de los poderes normativos de inteligibilidad cultural a los individuos-no-sujetos, la violencia y las lesiones psico-físicas, sociales, jurídicas y simbólicas impuestas a aquellos que no son reconocidos qua sujetos. Esta dimensión concreta de la violencia es la explotación del lazo original de interdependencia intersubjetiva y normativa. Esto último es particularmente interesante no sólo porque señala el fundamento metafísico-ontológico de la violencia efectiva, sino también porque indica la direccionalidad del proyecto ético-político de Butler, el cual, al menos tal como se formula en estos textos, tiene dos metas complementarias. Por un lado, reconocer la precariedad como condición

18 En este sentido debe comprenderse la apropiación que Butler realiza en Contingencia, hegemonía y universalidad ([2000] 2011, pp. 23-28) de la crítica que Hegel formula al formalismo kantiano en el Concepto previo de la Ciencia de la Lógica de la Enciclopedia, cuestión que aquí no repongo por falta de espacio.

19 El concepto de «precariedad» es propio de Marcos de guerra ([2009] 2010, p. 15); el de «desposesión» lo es de Desposesión ([2013] 2017, pp. 15-16, 37) y la idea de vulnerabilidad primaria es de Mecanismos ([1997] 2015, pp. 31-40), Vida precaria ([2004] 2009, pp. 57-73) y Cuerpos aliados ([2015] 2017, pp. 135-150). 
irreductible de la vida humana y configurar esquemas de inteligibilidad y reconocibilidad que contemplen su aprehensión. Por otro lado, minimizar la precaridad de manera responsable, tendiendo a disminuir progresivamente las diversas modalidades efectivas de violencia.

\section{Consideraciones FINALES}

En este trabajo se mostró que la concepción posthegeliana del reconocimiento de Butler consta de dos mediaciones, la mediación social y la normativa, que ponen de relieve dos determinaciones esenciales de la formación del sujeto a través del reconocimiento: la socialidad y la normatividad. Se mostró también que aquellas implican dos nociones de «sujeto», que tienen, al menos en términos débiles, caracteres neohegelianos. En un primer sentido, el Sujeto es una figura histórico-normativa que los individuos establecen mediante sus propias prácticas consuetudinarias hegemónicas, y también es la autoconciencia colectiva que una sociedad determinada alcanza en dicha figura. En un segundo sentido, el sujeto es el resultado de un proceso de formación, cuyo punto de cumplimiento radica en el reconocimiento intersubjetivo realizado en el seno de marcos culturales e institucionales que se estructuran en función de esquemas normativos de inteligibilidad y reconocibilidad subjetivas. Esto llevó, en tercer lugar, a desarrollar la sustituibilidad subjetiva y el carácter precario de la individualidad implicados en la concepción butleriana de la subjetividad.

Sin lugar a duda, la hermenéutica butleriana del Capítulo IV de la Fenomenología es susceptible de muchas objeciones, dado que toma un posicionamiento ajeno a la filosofía hegeliana. En primer lugar, como desarrollé en otro lugar (Abellón, 2020b), la Begierde se restringe a la Parte introductoria del capítulo y tiene un sentido muy restringido en la Fenomenología, limitándose a ser sólo la referencia negativa y meramente inmediata del algo vivo, diferenciándose de la categoría de «reconocimiento» y de las otras dos referencias negativas y mediadas que se exponen en la Sección A, el goce y el trabajo. En segundo lugar, como se mencionó, el cuerpo es una clave hermenéutica externa al Capítulo IV de la Fenomenología. La corporalidad se tematiza recién en el Capítulo V, donde Hegel define el cuerpo (Leib) no como una forma o figura (Gestalt) sino como la unidad del ser originario y determinado no formado (ungebildet) y del ser formado (gebildet) ([1807] 2010, 171-172). A diferencia de «Bildung», "Gestalt» no refiere a un proceso de formación o perfeccionamiento cultural, sino que denota una configuración ya acabada. Assalone (2013, pp. 53-56) y Malabou (2010, pp. $89,92)$ consideran oportuna la tesis de Butler según la cual el cuerpo estaría presupuesto en el Capítulo IV. En este sentido, la idea de Butler tendría el valor de problematizar una noción que parte de la Hegelforschung utiliza sin 
fundamentar, como son los casos de Álvarez (2010), Honneth (2010), Jenkins (2009) y Neuhouser (2009).20 Sin embargo, considero que, en este caso y por las razones aducidas, la hermenéutica de Butler carece de conexiones textuales rigurosas. En tercer lugar, su apropiación de la idea de Eticidad, realizada desde un claro lineamiento foucaultiano, sufre los problemas propios de toda lectura apropiativa: es parcial e introduce cuestiones externas a la obra. Además, su tratamiento de la concepción hegeliana del reconocimiento es sumamente reducido. Butler no considera la totalidad del movimiento del reconocimiento en la Fenomenología y tampoco recurre a las otras obras en las que Hegel se ocupa de este tema, lo que trae como consecuencia la desatención de estructuras fundamentales que hacen al movimiento del reconocimiento, como son la familia, el Estado y otras configuraciones institucionales.

Pero lo que me interesa aquí no es desarrollar in extenso el distanciamiento crítico de la lectura butleriana de Hegel -cuestión que requeriría otro tipo trabajo-, sino más bien subrayar que su concepción posthegeliana del reconocimiento y su concepción del sujeto concomitante son fundamentales para comprender la filosofía de Butler en general. El corpus butleriano es multifacético y examina un abanico variado de temáticas contemporáneas. Empero, una cuestión central que atraviesa la gran mayoría de sus trabajos es la problemática de la subjetividad. El problema del sujeto y de su proceso de formación constituyen un eje estructural y fundamental de su filosofía. A partir del examen de distintas temáticas, Butler intenta dilucidar los sentidos del sujeto que las sociedades contemporáneas elaboran, así como también los mecanismos que hacen a su producción, mantenimiento y posible desarticulación. Cada variable social analizada revela lógicas culturales peculiares, como son los casos del género y la sexualidad. Sin embargo, entiendo que, en su filosofía, hay una forma o modelo general de constitución subjetiva, que radica en la concepción posthegeliana del reconocimiento desarrollada. En este sentido, las múltiples variables que conforman al sujeto no constituyen, cada una de ellas, una lógica aislada, sino que forman parte de los diversos criterios normativos de los esquemas de inteligibilidad y normatividad subjetivas que median el reconocimiento a partir del cual los individuos devienen sujetos.

\section{REFERENCIAS BIBLIOGRÁFICAS}

ABELLÓN, M. (2020a): «Las mediaciones de la autoconciencia erótica. La hermenéutica butleriana del Capítulo IV de la Fenomenología del espiritu», 
Tópicos. Revista de Filosofía, 59, Universidad panamericana, México, pp. 153193.

ABELLÓN, M. (2020b): «La concepción erótica de la subjetividad en la Fenomenología del espíritu. Alcances y límite», Éndoxa, 45, Madrid, pp. 99-116.

ÁLVAREZ, E. (2010), «La autoconciencia: lucha, libertad y desventura», en F. Duque (ed.), Hegel. La odisea del espíritu. Madrid: Ediciones Pensamiento, pp. 85-108.

ALTHUSSER, L. (2012), «Ideología y aparatos ideológicos del Estado», en L. Althusser, Posiciones. Barcelona: Anagrama.

ASSALONE, E. (2013), "El cuerpo en el Capítulo IV de la Fenomenología del espíri$t u$ de Hegel. La lectura de Judith Butler», en L. Basso y P. Bedin (eds.), Estudios filosóficos en torno a la corporalidad. Convergencias y Divergencias. Actas de XII Jornadas nacionales. Agora philosophica. Buenos Aires: Biblioteca Electrónica de la Asociación Argentina de Investigaciones Éticas, pp. 48-57.

ASSALONE, E. (2015a), «Hegel normativista. La prioridad de la práctica, la autoconciencia como logro social y como sujeto de estados normativos en el Cap. IV de la Fenomenología del espíritu», Ideas y valores, LXIV (158), pp. 61-84.

ASSALONE, E. (2015b), «J. Butler y A. Honneth: convergencias y divergencias en torno a la relación entre la subjetividad, la normatividad, el reconocimiento y la exclusión, en el cruce entre la filosofía y la teoría social», en S. Barbosa, M. Santander y A. Florito Mutton (comps.), Actas de las VI Jornadas Nacionales de Antropología Filosófica. Buenos Aires: Universidad Nacional de Mar del Plata, pp. 42-46.

BOUTON, C. (2009), «Les apories de la lutte pour la reconnaissance. Hegel, Kojève, Butler», en F. Brugère y G. Le Blanc (eds.), Judith Butler. Trouble dans le sujet, trouble dans les normes. Paris: PUF, pp. 35-67.

BUTLER, J. [1987/1999] 2012: Sujetos del Deseo. Reflexiones hegelianas en la Francia del siglo XX. Trad. de E. L. Odriozola. Buenos Aires: Amorrortu.

BUTLER, J. [1997] 2015: Mecanismos psíquicos del poder. Teorías sobre la sujeción. Trad. de J. Cruz. Madrid: Cátedra.

BUTLER, J. [2000] 2001: El grito de Antígona. Trad. de E. Oliver, Barcelona: El Roure.

BUTLER, J., LACLAU, E. y ŽIŽEK, S. [2000] 2011: Contingencia, Hegemonía, Universalidad. Diálogos contemporáneos en la izquierda. Trad. de C. Sardoy y G. Homs. México: Fondo de Cultura Económica.

BUTLER, J. [2004] 2006: Deshacer el género. Trad. de P. Soley-Beltran. Barcelona: Paidós.

BUTLER, J. [2004] 2009: Vida precaria. El poder del duelo y la violencia. Trad. de F. Rodríguez, Buenos Aires: Paidós.

BUTLER, J. [2005] 2009: Dar cuenta de sí mismo. Violencia ética y responsabilidad. Trad. de H. Pons, Buenos Aires: Amorrortu.

BUTLER, J. [2009] 2010: Marcos de guerra. Las vidas lloradas. Trad. de B. Moreno, Buenos Aires: Paidós.

BUTLER, J. y MALABOU, C. 2010: Sois mon corps. Une lecture contemporaine de la domination et de la servitude chez Hegel. Montrouge: Bayard Éditions 
(2011: «You Be My Body for Me: Body, Shape, and Plasticity in Hegel's Phenomenology of Spirit», en S. Houlgate y M. Baur (eds.), A Companion to Hegel. West Sussex: Wiley-Blackwell, pp. 611-640.

BUTLER, J. [2013] 2017: Desposesión: lo performativo en lo político Trad. de Fernando Bogado, Buenos Aires: Eterna Cadencia.

BUTLER, J. [2015] 2016: Los sentidos del sujeto. Trad. de P. Kuffer, Barcelona: Herder, 2016.

BUTLER, J. [2015] 2017: Cuerpos aliados y lucha politica. Hacia una teoría performativa de la asamblea. Trad. de M. J. Viejo, Barcelona: Paidós, 2017.

CAVARERO, A. 1997: Tu che mi guardi, tu che mi racconti. Milàn: Giacomo Feltrinelli.

DAVIS, N. (2012), «Subjected Subjects? On Judith Butler's Paradox of Interpellation», Hypatia, 27-4, pp. 881-897.

DE LA MAZA, M. (2009), «El sentido del reconocimiento en Hegel», Revista Latinoamericana de Filosofía, XXXV, pp. 227-251.

DE LA MAZA, M. (2010), «Actualizaciones del concepto hegeliano de reconocimiento», Veritas, 23, pp. 67-94.

DÜSING, E. 1986: Intersubjektivität und Selbstbewusstsein. Köln: Verlag für Philosophie Dinter.

FOUCAULT, M. 1980: Power/Knowledge: Selected Interviews and Other Writings, 1972-77. New York: Pantheon.

FOUCAULT, M. 1986: Vigilar y castigar. Madrid: Siglo XXI.

FOUCAULT, M. 2002-2003: Historia de la sexualidad I-II. Buenos Aires: Siglo XXI.

HEGEL, G. W. F. [1807] 2010: Fenomenología del espírit. Trad. de Gómez Ramos, Ed. Bilingüe. Madrid: Abada (2007, Trad. de W. Roces, 2da. Ed., México: Fondo de Cultura Económica; 1997: Phenomenology of Spirit. Trad. de A. V. Miller, Oxford: Clarendon Press).

HONNET, A. (2010), «Von der Begierde zur Anerkennung; Hegels Begründung von Selbstbewußtsein», en A. Honneth, Das Ich im Wir. Studien zur Anerkennungstheorie. Berlin: Suhrkampp, pp. 15-32.

JENKINS, S. (2009), «Hegel's Concept of Desire», Journal of the History of Philosophy, 47-1, pp. 109-130.

KOJÈVE, A. [1947] 2006: La dialéctica del amo y el esclavo en Hegel. Trad. de J. J. Sebreli, Buenos Aires: Leviatán.

LOYD, M. 2007: Judith Butler. From Norms to Politics. Cambridge: Polity Press.

MALABOU, C. (2010), «Mais qu'est-ce que former le corps?», en J. Butler y C. Malabou, Sois mon corps. Montrouge: Bayard Éditions, pp. 85-96.

MALABOU, C. (2011), «Is Confession the Accomplishment of Recognition? Rousseau and the Unthought of Religion in the Phenomenology of Spirit», en C. Davis, C. Crokkett y S. Žižek (eds.), Hegel and The Infinite. Religion, Politics, and Dialectic. New York: Columbia University Press, pp. 19-30.

MILLS, C. (2015), «Undoing Ethics: Butler on Precarity, Opacity and Responsibility», en M. Lloyd (ed.), Butler and Ethics. Edinburgh: Edinburgh University Press, pp. 41-64. 
NEUHOUSER, F. (2009), «Desire, Recognition, and the Relation between Bondsman and Lord», en K. Westphal (Ed.), The Blackwell Guide to Hegel's Phenomenology. Oxford: Wiley Blackwell, pp. 37-54.

ONG-VAN-CUNG, K. (2010): «Reconnaissance et vulnérabilité. Honneth et Butler», Archives de Philosophie, 73, pp. 119-141.

ROCKMORE, T. 2005: Hegel, Idealism, and Analytic Philosophy. New Haven-London: Yale University Press.

RUSSON, J. 1997: The Self and Its Body in Hegel's Phenomenology of Spirit. Toronto: University of Toronto Press.

SABOT, P. (2011), «Attachement et relationnalité: Butler face à Hegel», Methodos, 11, pp. 1-20.

SIEP, L. 1979: Anerkennung als Prinzip der praktischen Philosophie. Friburgo-Munich: Felix Meiner.

SIEP, L. 2004: Konkrete Ethik, Frankfurt am Main: Suhrkamp.

SIEP, L. (2006), «Die Bewegung des Anerkennes in der Phänomenologie des Geistes», en D. Köhler y O. Pöggeler, G. W. F. Hegel, Phänomenologie des Geistes (Klassiker auslegen, 16), 2. Aufl. Berlin, pp. 107-127.

SIEP, L. 2010: Die Aktualität und Grenzen der Praktischen Philosophie Hegels. München: W. Fink.

SIEP, L. (2014), «Sobre la actualidad de la teoría del reconocimiento de Hegel», Cuadernos de filosofia, 63, pp. 5-22.

STARK, H. (2014), «Judith Butler'post-Hegelian ethics and the problem with recognition», Feminist Theory, 15-1, pp. 89-100.

TAYLOR, C. ([1992] 1997), «The politics of Recognition», en C. Taylor, Philosophical Arguments. Cambridge and London: Harvard University Press, pp. 225-256.

THEUNISSEN, M. (1982), «Die verdrängte Intersubjektivität in Hegels Philosophie des Rechts», en D. Henrich y R. Horstmann (eds.), Hegels Philosophie des Rechts. Die Theorie der Rechtsformen und ihre Logik. Stuttgart: Klett-Cotta.

Milton Abellón es Doctor en Filosofía por la Universidad de Buenos Aires, Investigador del Consejo Nacional de Investigaciones Científicas y Técnicas, y Docente de Ética en la Facultad de Filosofía y Letras de la Universidad de Buenos Aires.

Líneas de Investigación:

Recepciones contemporáneas de la Filosofía de Hegel, Filosofía Contemporánea, Ética y Antropología Filosófica.

Publicaciones recientes:

(2020): «Las mediaciones de la autoconciencia erótica. La hermenéutica butleriana del Capítulo IV de la Fenomenología del espíritu», Tópicos. Revista de Filosofía, 59, Universidad panamericana, México, pp. 153-193. 
(2020): «La concepción erótica de la subjetividad en la Fenomenología del espíritu. Alcances y límite», Éndoxa, 45, Madrid, pp. 99-116.

Correo electrónico: milton.abellon@gmail.com 